\title{
Bifurcation Problems for Generalized Beam Equations
}

\author{
Fosheng Wang \\ Department of Mathematics, Sichuan University, Chengdu 610064, China \\ Correspondence should be addressed to Fosheng Wang; fosheng321@163.com
}

Received 4 October 2014; Accepted 4 December 2014; Published 22 December 2014

Academic Editor: Ricardo Weder

Copyright (C) 2014 Fosheng Wang. This is an open access article distributed under the Creative Commons Attribution License, which permits unrestricted use, distribution, and reproduction in any medium, provided the original work is properly cited.

We investigate a class of bifurcation problems for generalized beam equations and prove that the one-parameter family of problems have exactly two bifurcation points via a unified, elementary approach. The proof of the main results relies heavily on calculus facts rather than such complicated arguments as Lyapunov-Schmidt reduction technique or Morse index theory from nonlinear functional analysis.

\section{Introduction and Main Results}

In physics, the vibration of an elastic beam, with length 1 and one endpoint hinged at $x=0$, which is compressed at the free edge $(x=1)$ by a force of intensity proportional to $\lambda>0$, is governed by the so-called beam equation

$$
\varphi^{\prime \prime}+\lambda \sin \varphi=0 \quad \text { in }(0,1)
$$

see [1]. The beam maintains its shape when the "force" $\lambda$ is sufficiently small, but it will buckle once $\lambda$ exceeds a certain value. In mathematics, the set of such values can be studied by exploiting the homogeneous Neumann boundary value problem:

$$
\begin{gathered}
\varphi^{\prime \prime}+\lambda \sin \varphi=0 \quad \text { in }(0,1), \\
\varphi^{\prime}(0)=\varphi^{\prime}(1)=0 .
\end{gathered}
$$

Before stating precisely the properties which we will explore in BVP (2), we embed this problem into a family of such boundary value problems; that is, we introduce the family of problems

$$
\begin{gathered}
\varphi^{\prime \prime}+\lambda h \circ \varphi=0 \quad \text { in }(a, b), \\
\varphi^{\prime}(a)=\varphi^{\prime}(b)=0,
\end{gathered}
$$

where $a, b \in \mathbb{R}$ with $a<b, \lambda$ belongs to a certain nonempty subset of $\mathbb{R}$, and $\varphi=\varphi(x)$ is the unknown; the function $h \in$
$C^{\infty}(\mathbb{R} ; \mathbb{R})$ satisfies the following: there exists an $l>0$ such that

(H1) $h(x+l)=-h(x)$, for all $x \in \mathbb{R}$,

(H2) $x_{0} \in \mathbb{R}$ for which

$$
\begin{gathered}
h^{\prime}\left(x_{0}\right)>0, \\
h\left(x_{0}+x\right)=-h\left(x_{0}-x\right)>0, \quad \forall x \in[0, l], \\
K:[0, l] \ni x \longmapsto \sqrt{\int_{x_{0}}^{x_{0}+x} h(t) \mathrm{d} t} \\
\in[0, \infty) \text { is a concave function. }
\end{gathered}
$$

Remark 1. We call the equation occurring in BVP (3) "generalized" beam equation; such equations are widely used to describe various physical phenomena.

Remark 2. It follows immediately from the hypothesis (5) that $h\left(x_{0}\right)=h\left(x_{0}+l\right)=0$ and from (H1) that $h$ is $2 l$-periodic.

Remark 3. It is easy to see that the function $h(x)=\sin x$, $x \in \mathbb{R}$, satisfies the hypotheses (H1)-(H2) with $l=\pi, x_{0}=0$.

Trivially, BVP (3) admits the trivial solution $\varphi=0$ for any $\lambda \in \mathbb{R}$. Here we are focused on the bifurcation theory for BVP (3). The bifurcation points are determined by eigenvalues associated with the differential operator $\varphi^{\prime \prime}+\lambda h \circ \varphi$. At such 
points, the number of solutions to (3) may change. However, very little further work has been done to determine whether the number of solutions changes at these points. In this paper, we give such a criterion for a class of nonlinear problems.

Theorem 4. Let $a, b, \lambda \in \mathbb{R}$ with $a<b$. Assume (H1)-(H2). Then

$$
\frac{ \pm \pi^{2}}{\left((b-a)^{2} h^{\prime}\left(x_{0}\right)\right)}
$$

are two bifurcation points for BVP (3). Besides, (3) has nonconstant solutions if and only if

$$
\pi^{2}<|\lambda|(b-a)^{2} h^{\prime}\left(x_{0}\right) .
$$

The proof of a bifurcation assertion of a nonlinear equation often has as ingredients such topological arguments as Krasnoselskii's and Rabinowitz's theorems on bifurcation. These arguments usually have the assumption that the algebraic multiplicity of the associated linear eigenvalue problem is odd; see [1-3] and the references therein. Since then, several authors have also attempted to remove such oddness assumption; see [1, 2, 4]. In particular, Ma and Wang [2] developed an elaborate algorithm to prove steady state bifurcation assertions concerning nonlinear equations; this algorithm does not assume the oddness of the algebraic multiplicity. See [5-13] for more studies on bifurcation problems. Our approach to prove Theorem 4 does not assume such parity condition on the algebraic multiplicity.

As a matter of fact, BVP (2) is a special case of SturmLiouville problem or boundary value problems for elliptic partial differential equations. Therefore, BVP (2), possibly in disguise, has been studied extensively in the literature for the existence of solutions satisfying certain prescribed properties, for qualitative properties of solutions, and so on; see [14-17] and the profound references cited therein.

The remainder of this paper is organized as follows. In Section 2 we introduce some nonlinear functional analysis and formulate the problem in a formal way, and in Section 3 we give the proof of Theorem 4 .

\section{The Existing Bifurcation Results for BVP (2)}

In this section, we mainly give a brief review of the existing results in the literature concerning bifurcation problems for BVP (2) which can be viewed as archetypes of bifurcation problems for BVP (3). Indeed, bifurcation problems for BVP (2) have been often provided as illustration examples to test the proposed abstract bifurcation-problems-solving method in the literature; see $[1,12,13,18]$.

In particular, Ma and Wang [18] proposed an abstract method which generalizes slightly the previous one obtained by Nirenberg [1]. In presenting their method, the authors fixed two Banach spaces $X$ and $Y$ for which $X$ embeds continuously and densely into $Y$. The abstract problem which they were concerned with reads

$$
L_{\lambda} u+G_{\lambda}(u)=0
$$

where $L_{\lambda}: X \rightarrow Y, \lambda \in \mathbb{R}$, is a family of bounded linear operators and $G_{\lambda}: X \rightarrow Y$ is a family of continuous mappings. They assumed the following.

(H3) $L_{\lambda}$ is in the form $L_{\lambda}=A+B_{\lambda}$ with $A$ as a linear topological isomorphism of $X$ onto $Y$ and $B_{\lambda}$ as compact linear operators; hence the spectrum of $L_{\lambda}$ consists of the exactly countably many eigenvalues $\left\{\beta_{k}(\lambda)\right\}$ (listed by algebraic multiplicities) of $L_{\lambda}$; there exists $\lambda_{0}$ for which

$$
\begin{gathered}
\beta_{1}\left(\lambda_{0}\right) \neq \beta_{j}\left(\lambda_{0}\right), \quad \beta_{j}\left(\lambda_{0}\right) \neq 0, \quad \forall j>1, \\
\beta_{j}\left(\lambda_{0}\right)<0, \quad \text { if } \lambda<\lambda_{0}, \\
\beta_{1}\left(\lambda_{0}\right)=0, \quad \text { if } \lambda=\lambda_{0}, \\
\beta_{j}\left(\lambda_{0}\right)>0, \quad \text { if } \lambda>\lambda_{0} .
\end{gathered}
$$

(H4) For any $\varepsilon>0$, there exists a $\delta>0$ such that

$$
\left\|G_{\lambda}(u)\right\|_{Y}<\varepsilon, \quad \forall u \in X \text { with }\|u\|_{X}<\delta, \quad \forall \lambda \in \mathbb{R} .
$$

$G_{\lambda}$ is analytic in the sense that

$$
G_{\lambda}=G_{\lambda}^{0}+\sum_{n=1}^{\infty} G_{\lambda}^{n}(\underbrace{u, u, \ldots, u}_{n \text { copies }})
$$

$G_{\lambda}^{n}$ is a continuous, symmetric $n$-form on $X$.

The precise problem with which they are concerned is whether there is a $\lambda_{0} \in \mathbb{R}$ given in such a way that if $u_{\lambda} \neq 0$ with $\lambda$ in a neighborhood of $\lambda_{0}$ is a collection of solutions to $\operatorname{BVP}(9)$, then

$$
u_{\lambda} \longrightarrow 0 \quad \text { in } X, \quad \text { as } \lambda \longrightarrow \lambda_{0}
$$

If there exists a $\lambda_{0}$ which satisfies the above requirements, then $\lambda_{0}$ is called a bifurcation point for nonlinear problem (9); also, problem (9) is said to bifurcate from $\lambda_{0}$.

Concerning (9), they proved the following.

Assume (H3)-(H4). Then $\lambda_{0}$ is a candidate bifurcation point of the nonlinear problem (9).

The proof of the above theorem provided in [18] utilizes such complicated methods as Lyapunov-Schmidt reduction method, Morse index theory, and so forth.

$\mathrm{Ma}$ and Wang [18] used the above theorem to obtain the bifurcation results for BVP (2). Indeed, they wrote firstly $X=\left\{u \in H^{2}(0,1) ; u^{\prime}(0)=u^{\prime}(1)\right\}, Y=L^{2}(0,1), L_{\lambda} u=$ $\left(d^{2} / d x^{2}\right) u-\lambda u$, and $G_{\lambda}(u)=\lambda \sin u-\lambda u$, thereby recasting BVP (2) into one of the forms (9), and secondly they solved this new bifurcation problem for BVP (2) by utilizing their abstract result.

Here we are tempted to use the results obtained in $\mathrm{Ma}$ and Wang [18] to solve the bifurcation problem for BVP (3); it is however obvious that the nonlinear reaction $\mathbb{R} \ni u \rightarrow$ $h(\sin u) \in \mathbb{R}$ precludes our application of such results. In the next section, we will analyze the bifurcation problem for BVP (3) in an elementary way. 


\section{Proof of the Main Results}

In this section we propose two lemmas and then prove Theorem 4 based on them. Various calculus theorems are employed in our proofs, and the elementary equality

$$
\left(f^{-1}\right)^{\prime}(f(t))=\frac{1}{f^{\prime}(t)}
$$

is also used repeatedly.

For the sake of convenience, we write

$$
H(x)=K^{2}(x)=\int_{x_{0}}^{x_{0}+x} h(t) \mathrm{d} t \quad(0 \leq x \leq l) .
$$

The function $H$ is strictly increasing on $[0, l], H(0)=0$, and $H^{\prime}(x)=h\left(x_{0}+x\right) . H$ is $2 \pi$-periodic because

$$
H(x+2 l)-H(x)=\int_{x}^{x+2 l} h(t) \mathrm{d} t=\int_{-l}^{l} h\left(x_{0}+t\right) \mathrm{d} t=0,
$$

due to Remark 2.

Lemma 5. The function $K$ is strictly increasing and differentiable on $[0, l]$, with derivative

$$
K^{\prime}(x)= \begin{cases}\frac{H^{\prime}(x)}{(2 \sqrt{H(x)})} & 0<x \leq l, \\ \sqrt{\frac{h^{\prime}\left(x_{0}\right)}{2}} & x=0 .\end{cases}
$$

Moreover,

$$
K^{\prime}\left(K^{-1}(y)\right)=\frac{1}{2 y\left(H^{-1}\right)^{\prime}\left(y^{2}\right)} \quad(0<y<\sqrt{H(l)}) .
$$

Proof. Since $K=\sqrt{H}$ and $H$ is positive on $(0, l]$, the value $K^{\prime}(x)$ with $x \in(0, l]$ can be derived directly from (15) and thus half of (17) is obtained. Consequently,

$$
K^{\prime}(0)=\lim _{x \downarrow 0} K^{\prime}(x)=\lim _{x \downarrow 0} \frac{H^{\prime}(x)}{2 \sqrt{H(x)}} .
$$

Noting that $H^{\prime} \geq 0$ on $[0, l]$, L'Hospital rule shows that

$$
\left(K^{\prime}(0)\right)^{2}=\lim _{x \downarrow 0} \frac{\left(H^{\prime}(x)\right)^{2}}{4 H(x)}=\lim _{x \downarrow 0} \frac{2 H^{\prime}(x) H^{\prime \prime}(x)}{4 H^{\prime}(x)}=\frac{h^{\prime}\left(x_{0}\right)}{2} .
$$

Thus the other half of (17) is obtained. A simple computation using (17) and (14) gives (18).

Lemma 6. Define

$$
p(t)=\int_{0}^{t} \frac{1}{\sqrt{H(t)-H(y)}} \mathrm{d} y \quad(0<t \leq l) .
$$

For $0<t<l$, the integral above converges, and the function $p$ is strictly increasing on $(0, l]$. Besides,

$$
\lim _{t \downarrow 0} p(t)=\frac{\pi}{\sqrt{2 h^{\prime}\left(x_{0}\right)}} .
$$

Proof. Changing of variable $y=H^{-1}(u H(t))$ in the integral, we get

$$
\int_{0}^{t} \frac{1}{\sqrt{H(t)-H(y)}} \mathrm{d} y=\int_{0}^{1} \frac{\sqrt{H(t)}}{\sqrt{1-u}} \cdot\left(H^{-1}\right)^{\prime}(u(H(t))) \mathrm{d} u
$$$$
(0<t \leq l) .
$$

By the dominated convergence theorem and (18), the integrand of the right side above equals

$$
\frac{1}{2 \sqrt{u(1-u)} \cdot K^{\prime}\left(K^{-1}(\sqrt{u(H(t))})\right)},
$$

and thus

$$
p(t)=\int_{0}^{1} \frac{1}{2 \sqrt{u(1-u)} \cdot K^{\prime}\left(K^{-1}(\sqrt{u(H(t))})\right)} \mathrm{d} u .
$$

The function $1 /\left(K^{\prime} \circ K^{-1}\right)$ is strictly increasing on $(0$, $\sqrt{H(l)}]$ because $K^{-1}$ is strictly increasing and $K^{\prime}$ is strictly decreasing by our assumption (6). Hence value (24) increases as $t$ does. Note that $\sqrt{u(H(t))} \leq \sqrt{H(t)}$ for $u \in[0,1]$. If $t \in(0, l)$, then

$$
\begin{aligned}
p(t) & =\int_{0}^{1} \frac{1}{2 \sqrt{u(1-u)} \cdot K^{\prime}\left(K^{-1}(\sqrt{u(H(t))})\right)} \mathrm{d} u \\
& \leq \int_{0}^{1} \frac{1}{2 \sqrt{u(1-u)}} \cdot \frac{1}{K^{\prime}\left(K^{-1}(\sqrt{H(t)})\right)} \mathrm{d} u \\
& =\frac{\pi}{2 K^{\prime}\left(K^{-1}(\sqrt{H(t)})\right)}<\infty .
\end{aligned}
$$

Hence $p$ is real valued and (25) implies that $p$ is strictly increasing on $(0, l]$. Finally, equalities (25), (17) and the dominated convergence theorem show that

$$
\begin{aligned}
\lim _{t \downarrow 0} p( & t) \\
& =\lim _{t \downarrow 0} \int_{0}^{1} \frac{1}{2 \sqrt{u(1-u)} \cdot K^{\prime}\left(K^{-1}(\sqrt{u(H(t))})\right.} \mathrm{d} u \\
& =\int_{0}^{1} \frac{1}{2 \sqrt{u(1-u)} \cdot K^{\prime}\left(K^{-1}(0)\right)} \mathrm{d} u \\
& =\frac{\pi}{2 \sqrt{h^{\prime}\left(x_{0}\right)}} .
\end{aligned}
$$

The proof is complete.

Proof of Theorem 4. Assume $\varphi$ is a nonconstant solution of (3). The set $\left\{x \in[a, b]: \varphi^{\prime}(x) \neq 0\right\}$ is open and nonempty in $[a, b]$ and thus is a disjoint union of open intervals (provided $[a, x)$ and $(x, b]$ for $x \in(a, b)$ are viewed as "open intervals" 
in $[a, b])$. Let $\left(a_{0}, b_{0}\right)$ be such an open interval. Then $\varphi$ is nonconstant on $\left[a_{0}, b_{0}\right]$ and $\varphi^{\prime}\left(a_{0}\right)=\varphi^{\prime}\left(b_{0}\right)$. We will show that $b_{0}-a_{0}>\pi / \sqrt{|\lambda| h^{\prime}\left(x_{0}\right)}$, which is equivalent to (8).

Since differentiable functions have intermediate value property and $\varphi^{\prime}(x) \neq 0$ on $\left(a_{0}, b_{0}\right)$, we may assume $\varphi^{\prime}>0$ on $\left(a_{0}, b_{0}\right)$ without loss of generality. So $\varphi$ is strictly increasing on $\left[a_{0}, b_{0}\right]$ with inverse function $g=\varphi^{-1}$ defined on $\left[\varphi\left(a_{0}\right), \varphi\left(b_{0}\right)\right]$. A simple computation using (14) and the chain rule for differentiation shows that

$$
\begin{aligned}
& \frac{d\left(\left(1 / g^{\prime}(y)\right)^{2}\right)}{d y} \\
& =\frac{d\left(\left(\left(g^{-1}\right)^{\prime}(g(y))\right)^{2}\right)}{d y} \\
& =2\left(g^{-1}\right)^{\prime}(g(y)) \cdot\left(g^{-1}\right)^{\prime \prime}(g(y)) \cdot g^{\prime}(y) \\
& =2\left(g^{-1}\right)^{\prime \prime}(g(y)) .
\end{aligned}
$$

Condition (3) means

$$
\begin{gathered}
\left(g^{-1}\right)^{\prime \prime}(g(y))+\lambda h(y)=0 \quad\left(\varphi\left(a_{0}\right) \leq y \leq \varphi\left(b_{0}\right)\right), \\
\lim _{y \downarrow g\left(\varphi\left(a_{0}\right)\right)} g^{\prime}(y)=\lim _{y \uparrow g\left(\varphi\left(b_{0}\right)\right)} g^{\prime}(y)=+\infty .
\end{gathered}
$$

Integrating both sides of (29) and using (28), we get that

$$
\frac{1}{2\left(g^{\prime}(y)\right)^{2}}+\lambda H(y)=C_{1} \quad\left(\varphi\left(a_{0}\right)<y<\varphi\left(b_{0}\right)\right),
$$

with constant $C_{1}$. We assume $\lambda>0$ without loss of generality. Then

$$
g^{\prime}(y)=\frac{1}{\sqrt{2 \lambda}} \cdot \frac{1}{\sqrt{C_{2}-H(y)}} \quad\left(\varphi\left(a_{0}\right)<y<\varphi\left(b_{0}\right)\right)
$$

with $C_{2}=C_{1} / \lambda$. Consequently,

$$
H(y)<C_{2} \quad\left(\varphi\left(a_{0}\right)<y<\varphi\left(b_{0}\right)\right) .
$$

As the properties of $H$ stated before, the function $y \mapsto$ $H(y)$ is with period $2 l$, decreases on $[-l, 0]$, and increases on $[0, l]$. Equalities (30) and (32) yield that

$$
H(y) \longrightarrow C_{2} \quad\left(y \downarrow \varphi\left(a_{0}\right) \text { or } y \uparrow \varphi\left(b_{0}\right)\right) .
$$

This together with (33) and the properties of $H$ shows that there exists $t \in(0, l]$ and $z \in \mathbb{Z}$ such that $C_{2}=H\left(x_{0}+t\right)$, $\varphi\left(a_{0}\right)=x_{0}+2 l z-t$, and $\varphi\left(b_{0}\right)=x_{0}+2 l z+t$. Consequently,

$$
\begin{aligned}
b_{0}-a_{0} & =g\left(\varphi\left(b_{0}\right)\right)-g\left(\varphi\left(a_{0}\right)\right) \\
& =g\left(\varphi\left(b_{0}\right)-2 l z\right)-g\left(\varphi\left(a_{0}\right)-2 l z\right) \\
& =g\left(x_{0}+t\right)-g\left(x_{0}-t\right) \\
& =\int_{-t}^{t} g^{\prime}\left(x_{0}+y\right) \mathrm{d} y .
\end{aligned}
$$

Since $H$ is an even function, it follows from (32) that

$$
\begin{aligned}
b_{0}-a_{0} & =\frac{1}{\sqrt{2 \lambda}} \int_{-t}^{t} \frac{1}{\sqrt{H(t)-H(y)}} \mathrm{d} y \\
& =\sqrt{\frac{2}{\lambda}} \int_{0}^{t} \frac{1}{\sqrt{H(t)-H(y)}} \mathrm{d} y \\
& =\sqrt{\frac{2}{\lambda}} p(t) .
\end{aligned}
$$

Hence

$$
b-a \geq b_{0}-a_{0}=\sqrt{\frac{2}{\lambda}} p(t)>\lim _{t \downarrow 0} \sqrt{\frac{2}{\lambda}} p(t)=\frac{\pi}{\sqrt{\lambda h^{\prime}\left(x_{0}\right)}},
$$

which implies (8), and thus half of Theorem 4 is proved.

For the other half, we assume without loss of generality that $\lambda>0$, and assume (8) holds; that is,

$$
b-a \geq \frac{\pi}{\sqrt{\lambda h^{\prime}\left(x_{0}\right)}} .
$$

And we show that (2) has a nonconstant solution.

First, it follows from (27) that there exits $t_{0}>0$ such that

$$
\sqrt{\frac{2}{\lambda}} p\left(t_{0}\right)<b-a .
$$

Let

$$
b_{1}=a+\sqrt{\frac{2}{\lambda}} p\left(t_{0}\right)<b .
$$

Define a continuous function $g_{0}$ on $\left[-t_{0}, t_{0}\right]$ by $g\left(-t_{0}\right)=a$ and

$$
g_{0}^{\prime}(y)=\frac{1}{\sqrt{2 \lambda}} \cdot \frac{1}{\sqrt{H\left(t_{0}\right)-H(y)}} \quad\left(-t_{0}<y<t_{0}\right) .
$$

Definition (21) yields

$$
\begin{aligned}
g\left(t_{0}\right) & =a+\int_{-t_{0}}^{t_{0}} g_{0}^{\prime}(t) \mathrm{d} t \\
& =a+2 \frac{1}{\sqrt{2 \lambda}} \cdot \int_{0}^{t_{0}} \frac{1}{\sqrt{H\left(t_{0}\right)-H(y)}} \mathrm{d} t \\
& =a+\sqrt{\frac{2}{\lambda}} p\left(t_{0}\right) \\
& =b_{1} .
\end{aligned}
$$

Besides,

$$
\frac{1}{2\left(g_{0}^{\prime}(y)\right)^{2}}+\lambda H(y)=\lambda H\left(t_{0}\right) \quad\left(-t_{0}<y<t_{0}\right) .
$$


Differentiating both sides of (45) and using (28), we get

$$
\left(g_{0}^{-1}\right)^{\prime \prime}\left(g_{0}(y)\right)+\lambda h(y)=0 \quad\left(-t_{0} \leq y \leq t_{0}\right) .
$$

From (43), it follows that

$$
\lim _{y \downarrow-t_{0}} g_{0}^{\prime}(y)=\lim _{y \uparrow t_{0}} g^{\prime}(y)=+\infty \text {. }
$$

Define $\varphi_{0}=g_{0}^{-1}$. Then (44) and (45) say that

$$
\begin{gathered}
\varphi_{0}^{\prime \prime}(x)+\lambda h\left(\varphi_{0}(x)\right)=0 \quad\left(a<x<b_{1}\right), \\
\varphi_{0}^{\prime}(a)=\varphi_{0}^{\prime}\left(b_{1}\right)=0 .
\end{gathered}
$$

From (46) and assumption (H1), we see that

$$
\lim _{x \uparrow b_{1}} \varphi_{0}^{\prime \prime}(x)=\lim _{x \uparrow b_{1}}-\lambda h\left(\varphi_{0}(x)\right)=\lim _{x \uparrow t_{0}}-\lambda h(y)=h\left(t_{0}\right)=0,
$$

and similarly $\lim _{x \downarrow a} \varphi_{0}^{\prime \prime}(x)=0$. By defining $\varphi_{0}(x)=\varphi_{0}\left(b_{1}\right)$ when $b_{1}<x \leq b$, the function $\varphi_{0}$ is extended and thus defined on $[a, b]$ such that

$$
\begin{gathered}
\varphi_{0}^{\prime \prime}(x)+\lambda h\left(\varphi_{0}(x)\right)=0 \quad(a \leq x \leq b), \\
\varphi_{0}^{\prime}(a)=\varphi_{0}^{\prime}(b)=0 .
\end{gathered}
$$

The proof is complete.

Example 7 (BVP (2) revisited). Again we are concerned with BVP (2); namely,

$$
\begin{gathered}
\varphi^{\prime \prime}+\lambda \sin \varphi=0 \quad \text { in }(a, b), \\
\varphi^{\prime}(a)=\varphi^{\prime}(b)=0 .
\end{gathered}
$$

The problem has nonconstant solutions if and only if ( $b-$ a) $)^{2}|\lambda|<\pi^{2}$. Indeed, we see that

$$
\sin (x+\pi)=-\sin x \quad(x \in \mathbb{R})
$$

and, for some $x_{0} \in \mathbb{R}$,

$$
\begin{gathered}
0<\sin x=-\sin (-x) \quad(0<x<\pi), \\
h^{\prime}(0)>0, \\
x \longmapsto \sqrt{\int_{0}^{x} \sin t \mathrm{~d} t} \text { is a concave function on }[0, \pi] .
\end{gathered}
$$

Note that (52) is trivial since

$$
\sqrt{\int_{0}^{x} \sin t \mathrm{~d} t}=\sqrt{1-\cos x}=\sqrt{2} \cos \frac{x}{2} \quad(0 \leq x \leq \pi) .
$$

Remark 8. In this paper, we have solved a class of bifurcation problems for Neumann boundary value problems for semilinear elliptic equations; namely,

$$
\begin{gathered}
\varphi^{\prime \prime}+\lambda h \circ \varphi=0 \quad \text { in }(a, b), \\
\varphi^{\prime}(a)=\varphi^{\prime}(b)=0 ;
\end{gathered}
$$

the governing equation occurring in this boundary value problem generalizes the classical beam equation in the sense that the nonlinear interaction assumes the form $\mathbb{R} \ni u \mapsto$ $h(\sin u) \in \mathbb{R}$ instead of $\mathbb{R} \ni u \mapsto \sin u \in \mathbb{R}$. What is more important is that the bifurcation problem for the classical beam equation can be solved using abstract bifurcation theorems in nonlinear analysis, while the generalized beam equations can not be. We provided a unified approach to understand this class of problems. Indeed, our method is quite general and very elementary.

It is worthwhile to mention that bifurcation problems associated with beam equations other than the type (3) have been extensively studied; see [19-22] and the profound references cited therein. The approaches frequently used in the literature are quite different from ours and have as foundations much advanced, complicated knowledge in functional analysis.

\section{Conflict of Interests}

The author declares that there is no conflict of interests regarding the publication of this paper.

\section{Acknowledgments}

The author is grateful to the anonymous referees for their valuable suggestions.

\section{References}

[1] L. Nirenberg, Topics in Nonlinear Functional Analysis, vol. 6 of Courant Lecture Notes in Mathematics, New York University Courant Institute of Mathematical Sciences, New York, NY, USA, 2001, Chapter 6 by E. Zehnder, Notes by R. A. Artino, Revised reprint of the 1974 original.

[2] T. Ma and S. Wang, "Bifurcation of nonlinear equations: I. Steady state bifurcation," Methods and Applications of Analysis, vol. 11, no. 2, pp. 155-178, 2004.

[3] T. Ma and S. Wang, "Bifurcation of nonlinear equations. II. Dynamic bifurcation," Methods and Applications of Analysis, vol. 11, no. 2, pp. 179-209, 2004.

[4] M. A. Krasnoselskii, Topological Methods in the Theory of Nonlinear Integral Equations, translated by A. H. Armstrong and edited by J. Burlak, Pergamon Press, New York, NY, USA, 1964.

[5] S. N. Chow and J. K. Hale, Methods of Bifurcation Theory, vol. 251 of rundlehren der Mathematischen Wissenschaften [Fundamental Principles of Mathematical Science], Springer, New York, NY, USA, 1982.

[6] D. Henry, Geometric Theory of Semilinear Parabolic Equations, Lecture Notes in Mathematics, Springer, New York, NY, USA, 1981.

[7] T. Kato, Perturbation Theory for Linear Operators, Classics in Mathematics, reprint of the 1980 edition, Springer, Berlin, Germany, 1995.

[8] T. Ma and S. Wang, "Structure of 2D incompressible flows with the DIRichlet boundary conditions," Discrete and Continuous Dynamical Systems Series B, vol. 1, no. 1, pp. 29-41, 2001.

[9] T. Ma and S. Wang, "Structural classification and stability of divergence-free vector fields," Physica $D$, vol. 171, no. 1-2, pp. 107-126, 2002. 
[10] T. Ma and S. Wang, "Attractor bifurcation theory and its applications to Rayleigh-Bénard convection," Communications on Pure and Applied Analysis, vol. 2, no. 4, pp. 591-599, 2003.

[11] T. Ma and S. Wang, "Dynamic bifurcation and stability in the RayleighBénard convection," Communications in Mathematical Sciences, vol. 2, no. 2, pp. 159-183, 2004.

[12] T. Ma and S. Wang, Bifurcation Theory and Applications, vol. 53 of World Scientific Series on Nonlinear Science. Series A: Monographs and Treatises, World Scientific Publishing Co. Pte. Ltd., Hackensack, NJ, Hackensack, NJ, USA, 2005.

[13] T. Ma and S. Wang, Geometric Theory of Incompressible Flows with Applications to Fluid Dynamics, Mathematical Surveys and Monographs, American Mathematical Society, Providence, RI, USA, 2005.

[14] H. Berestycki, "On some nonlinear Sturm-Liouville problems," Journal of Differential Equations, vol. 26, no. 3, pp. 375-390, 1977.

[15] G. Birkhoff, A Source Book in Classical Analysis, Harvard University Press, Cambridge, Mass, USA, 1973.

[16] P. H. Rabinowitz, "Nonlinear Sturm-Liouville problems for second order ordinary differential equations," Communications on Pure and Applied Mathematics, vol. 23, pp. 939-961, 1970.

[17] A. Zettl, Sturm-Liouville Theory, vol. 121 of Mathematical Surveys and Monographs, American Mathematical Society, Providence, RI, USA, 2005.

[18] T. Ma and S. Wang, Stability and Bifurcation Problems for Nonlinear Evolution Equations, Science Press, Beijing, China, 2007, (Chinese).

[19] M. A. Abdul Hussain, "Bifurcation solutions of elastic beams equation with small perturbation," International Journal of Mathematical Analysis, vol. 3, no. 17-20, pp. 879-888, 2009.

[20] J. Berkovits, "On the bifurcation of large amplitude solutions for a system of wave and beam equations," Nonlinear Analysis: Theory, Methods \& Applications, vol. 52, no. 1, pp. 343-354, 2003.

[21] S. Lenci, G. Menditto, and A. M. Tarantino, "Homoclinic and heteroclinic bifurcations in the non-linear dynamics of a beam resting on an elastic substrate," International Journal of NonLinear Mechanics, vol. 34, no. 4, pp. 615-632, 1999.

[22] C. Polymilis, C. Skokos, G. Kollias, G. Servizi, and G. Turchetti, "Bifurcations of beam-beam like maps," Journal of Physics A: Mathematical and General, vol. 33, no. 5, pp. 1055-1064, 2000. 


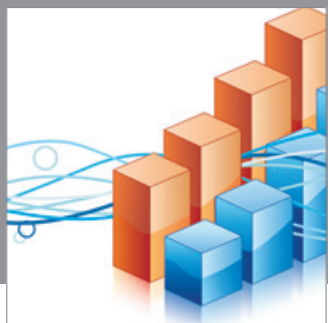

Advances in

Operations Research

mansans

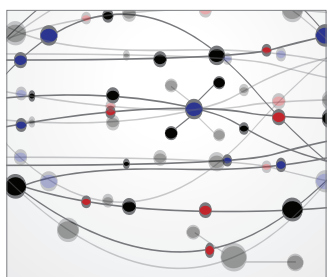

The Scientific World Journal
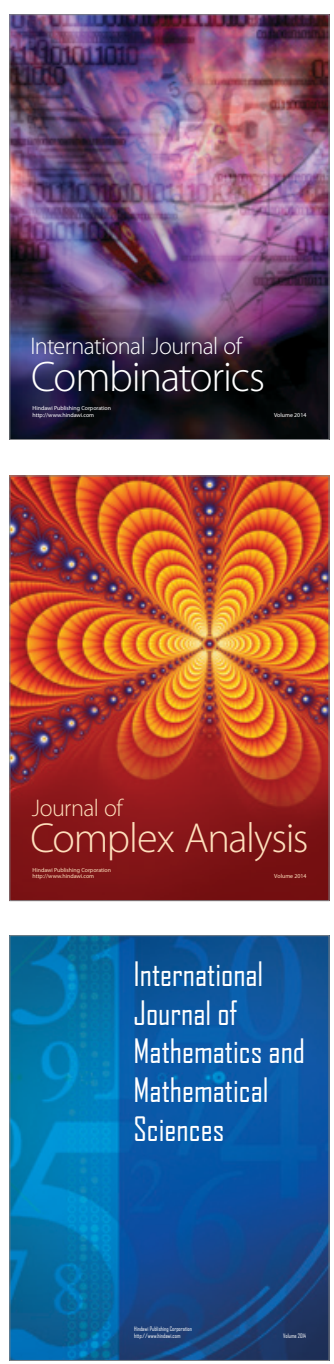
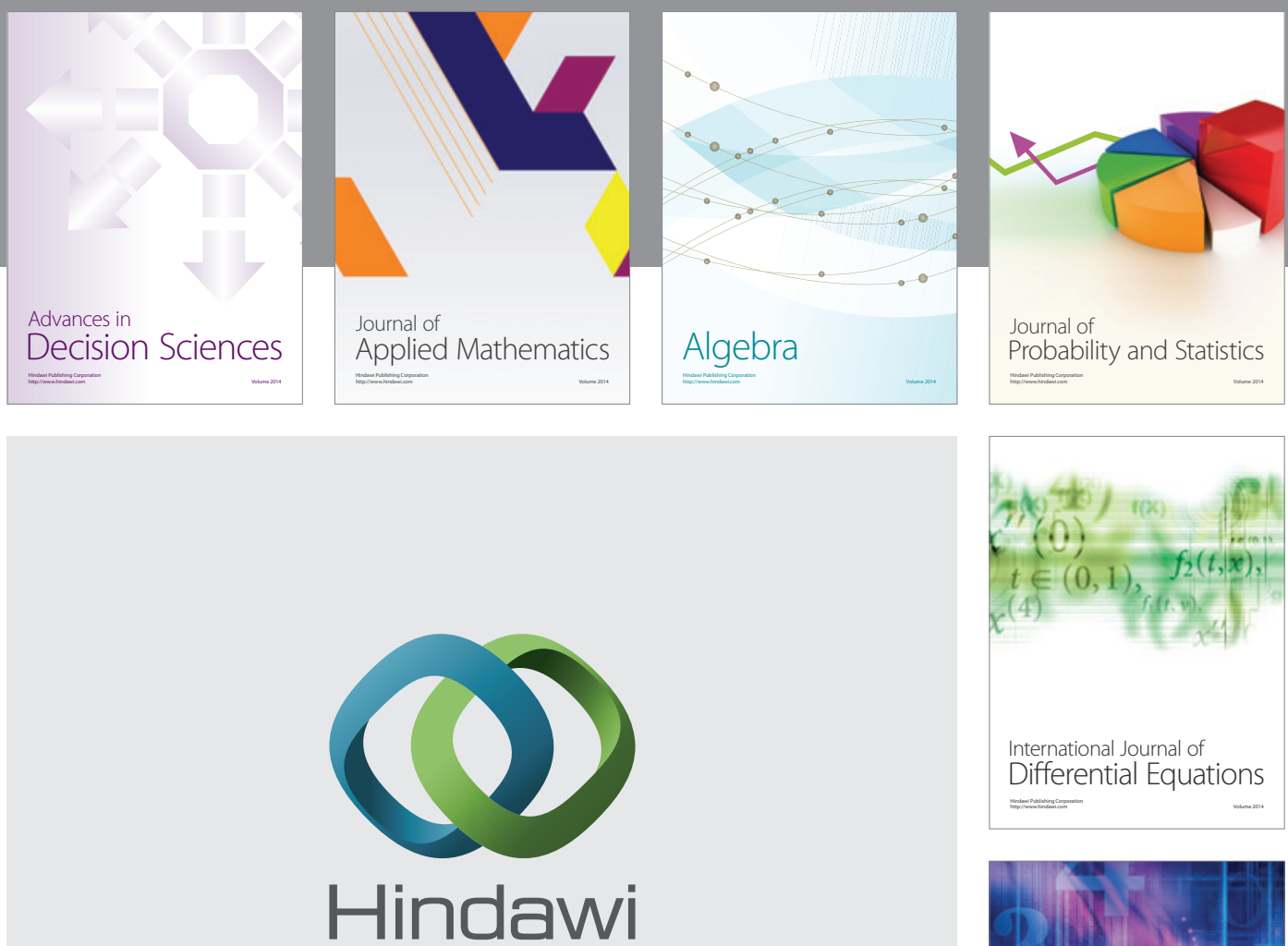

Submit your manuscripts at http://www.hindawi.com
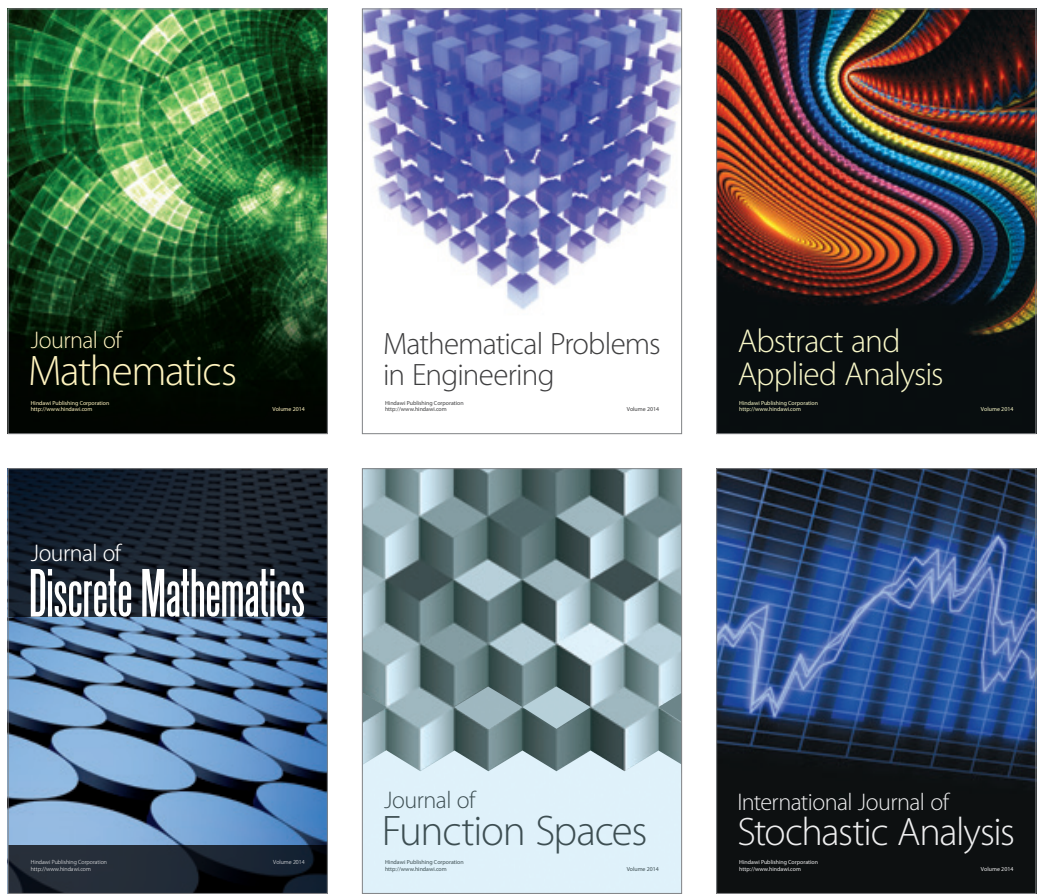

Journal of

Function Spaces

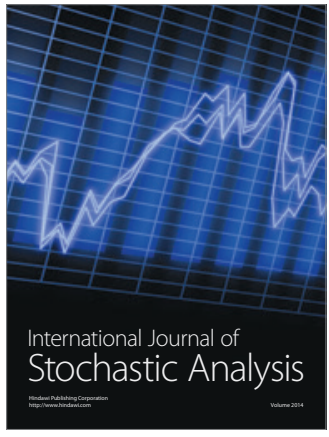

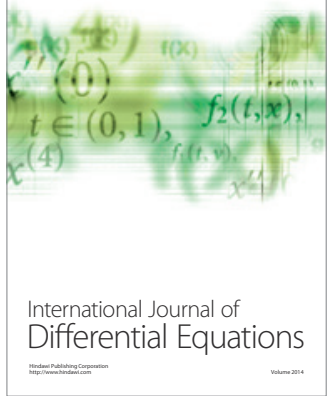
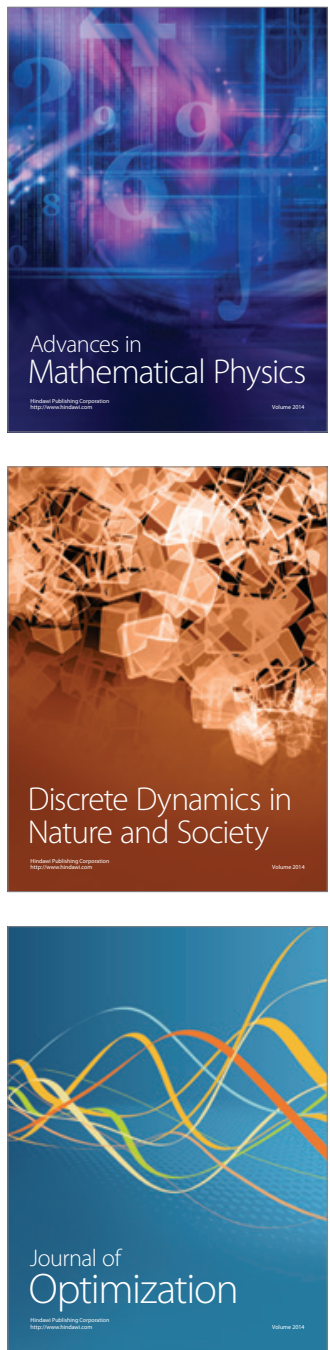